\title{
A symptomatic multiseptate gallbladder without gallstones
}

\author{
Damien Dousse $^{1 *}$, Laura Marcu ${ }^{2}$ and Francesco Martini ${ }^{1}$ \\ ${ }^{1}$ Department of Digestive Surgery, Joseph Ducuing Hospital, Toulouse, France \\ ${ }^{2}$ Department of Radiology, Joseph Ducuing Hospital, Toulouse, France
}

\begin{abstract}
\section{Case report}

A 30-year-old woman with no significant history, presented with recurrent pain in the right hypochondrium after rich meals since several years. The blood tests showed no abnormality, with normal inflammation markers and normal hepatic tests. Abdominal ultrasonography (Figure 1) found a multiseptate gallbladder (MSG) showing multiple transverse and non-vascularized septa, in the absence of dilatation or parietal thickening; no gallstone was found. The patient underwent laparoscopic cholecystectomy. Intraoperative cholangiography showed no abnormality. The pathological assessment confirmed the diagnosis of MSG (Figure 2). At the last control, three months after surgery, the patient was asymptomatic.
\end{abstract}

Multiseptate Gallbladder is a rare congenital abnormality, which can be totally asymptomatic or responsible for biliary pain in the absence of cholelithiasis or cholecystitis. In case of persistent symptomatology, cholecystectomy represent the treatment of choice.

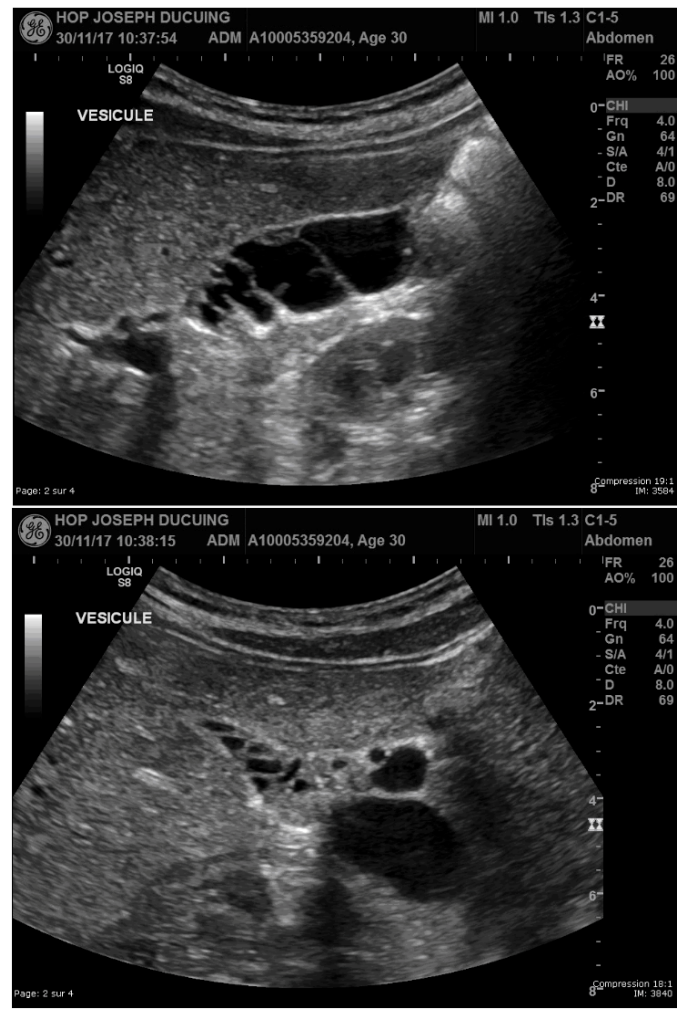

Figure 1. Ultrasound image of a multiseptate gallbladder.

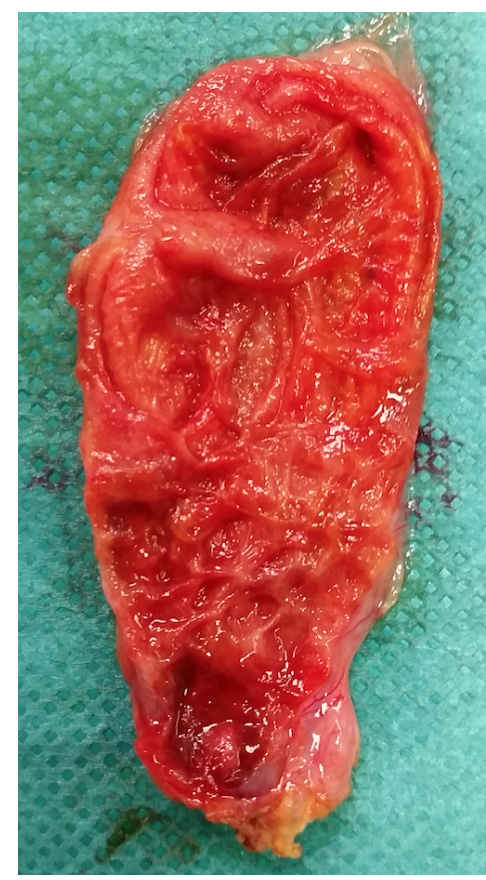

Figure 2. Image of a multiseptate gallbladder operative piece after opening along its major axis.

\section{Discussion and conclusion}

MSG is a rare congenital abnormality, most commonly found in children [1], in which the gallbladder is divided into several compartments of different sizes by intraluminal septa. Compartments are in communication with each other. Septa, covered by a typical cylindrical epithelium, can be partial or complete [2]. MSG may

Correspondence to: Damien Dousse, Department of Digestive Surgery, Joseph Ducuing Hospital, Toulouse, France, Tel: 00-86-23-46751087; Fax: + 3376276 61 30; E-mail: damien.dousse1@orange.fr

Key words: multiseptate gallbladder; cholecystectomy; biliary colic

Received: March 15, 2018; Accepted: April 03, 2018; Published: April 06, 2018 
be asymptomatic or may cause recurrent pain in the right upper abdominal quadrant, without abnormality of blood tests and without complications (sludge, gallstones, or cholecystitis) [1-3]. The literature concerning this rare condition is scanty with just a few case reports. Since regression of symptoms has been reported after cholecystectomy, surgical treatment is likely to be justified in case of persistent symptomatology [1-3].

\section{References}

1. Wanaguru D, Jiwane A, Day AS, Adams S (2011) Multiseptate Gallbladder in an Asymptomatic Child. Case Rep Gastrointest Med: 470658. [Crossref]

2. Geremia P, Tomà P, Martinoli C, Camerini G, Derhi LE (2013) Multiseptate gallbladder Clinical and ultrasonographic follow-up for 12 years. J Pediatr Surg 48: E25-E28. [Crossref]

3. Karaca T, Yoldas O, Bilgin BC, Bilgin S, Evcik E, et al. (2011) Diagnosis and Treatment of Multiseptate Gallbladder with Recurrent Abdominal Pain. Case Rep Med: 162853. [Crossref]

Copyright: $\odot 2018$ Dousse D. This is an open-access article distributed under the terms of the Creative Commons Attribution License, which permits unrestricted use, distribution, and reproduction in any medium, provided the original author and source are credited. 